\title{
Embodied multi-modal communication from the perspective of activity theory
}

\author{
Julian Williams
}

Published online: 13 November 2008

(C) Springer Science + Business Media B.V. 2008

\begin{abstract}
I begin by appreciating the contributions in the volume that indirectly and directly address the questions: Why do gestures and embodiment matter to mathematics education, what has understanding of these achieved and what might they achieve? I argue, however, that understanding gestures can in general only play an important role in 'grasping' the meaning of mathematics if the whole object-orientated 'activity' is taken into account in our perspective, and give examples from my own work and from this Special Issue. Finally, I put forward the notion of a 'threshold' moment, where seeing and grasping at the nexus of two or more activities often seem to be critical to breakthroughs in learning.
\end{abstract}

Keywords Activity theory $\cdot$ Unit of analysis $\cdot$ Pedagogy $\cdot$ Threshold moment

\section{Preface}

A large dog sat on the floor at the bar next to his owner, balefully eyeing me and other customers. I locked gaze with him and his sad-looking eyes responded; they suggested to me that he had been sitting there too long. I wondered if this was all in my imagination: do dogs speak to humans through their eyes? As I thought about it and about writing this paper, I laughed. The dog looked away; but then after a few moments he looked back at me, apparently to see if I was still watching him. The dog yawned, a huge gaping yawn that seemed to go on forever, and I felt impelled to yawn myself, and then I actually did yawn ${ }^{1}$.

Do humans and dogs communicate non-verbally? I recall a dog that, when its owner returned from work, would fetch the lead in its teeth to show him, drop it at his feet, and then run to the front door. Cats on the other hand are very different, at least in their facial expressions - perhaps this is due to a different co-evolution of the two species with human

\footnotetext{
${ }^{1}$ On dogs and humans empathy seen in yawning: http://www.guardian.co.uk/science/2008/aug/06/ animalbehaviour

J. Williams $(\bowtie)$

University of Manchester, Manchester, UK

e-mail: julian.williams@manchester.ac.uk
} 
cultures. Still, cats seem to know how to demand attention, food and comfort from humans quite effectively. In return, according to reports from psychological research, a pet is more stress-relieving for humans than a spouse ${ }^{2}$.

Scholars of human non-verbal communication used to claim that up to $90 \%$ of communicable information is non-verbally signalled, and teacher educators have sometimes tried to help teachers, especially in initial training, to take some control of their non-verbal behaviours when communicating in classrooms. The problem, of course, is that non-verbal behaviour largely operates below the conscious level and becomes different when made the focus of attention: if you ask a small child how it is that they tell their legs to walk alternately left then right, you may do damage. (I once tried this, and the small child looked to the left leg, then the right,... and then tripped.)

And yet, that is the interesting and crucial point about embodied communication: that it contributes so much, but at a level generally below the conscious, at the operational level. This is why, I believe, gestures are not the appropriate unit of analysis if 'meaning' is the focus of inquiry. As I will argue, Activity Theory suggests that analysis of meaning implies at least a three-level analysis: at operational, action, and activity levels. The essence of meaning is held to be found at the level of social, culturally mediated, collective, uniquely human, 'activity', presumably not found in pre-human or animal societies (Leont'ev 1978).

\section{This issue - an appreciation and hypotheses}

I also recall, as did Luis Radford (2008a) that at the PME symposium on gestures and mathematics education in Melbourne, 2005, we were asked by a sceptical critic: "What is all this about gestures and mathematics? What is the point?" I was at first puzzled: surely learning mathematics is all about communication, and any cursory look at communication leads one to the 'non-verbal', including gestures. But then, if one places oneself in the questioner's mindset, one can reasonably ask: what has the study of gestures achieved for mathematics education, and what might it achieve?

Let us consider how far the studies reported in this special issue have gone towards answering these questions. First, Roth and Thom (2008), Radford (2008a), and Nemirovski and Ferrara's (2008) three papers present arguments and illustrative evidence for a sensuous, embodied and material view of the formation of mathematical objects, conceptions and imaginations, respectively. These should leave our doubting critic with a sense that much of mathematics learning, at least, and possibly some essentials of mathematics learning cannot be other than sensuous and embodied. They collectively suggest a further generalisation: that any dichotomisation of the 'mental' and the 'embodied' is generally and in principle false.

Second, the three papers by Arzarello, Paulo, Robutti, and Sabena (2008), by Edwards (2008) and by Maschietto and Bartolini Bussi (2008) provide rich descriptions of gestures in multi-modal communications, involving learners and teachers in mathematical activity in classrooms (Arzarello et al. 2008; Maschietto \& Bartolini Bussi 2008) and by studentteachers in talk about mathematics (Edwards 2008). The other three also contribute to this abundance of evidence of the potential for 'reading' classroom activity through the lens of embodied communications. The six papers collectively add some analytical conceptions that are shown to clarify and help us to understand richly described, illustrative events: the classification of gestures by their functions (indexical, iconic, symbolic etc., in Edwards

${ }^{2}$ http://www.avma.org/onlnews/javma/nov02/021101m.asp 
2008); the 'synchronic and diachronic' in analysis of 'semiotic bundles' and the 'semiotic game' (Arzarello et al. 2008); and additionally the inclusion of material pedagogic artefacts and inscriptions in the understanding of these multi-modalities (Maschietto \& Bartolini Bussi 2008). These build on previous work by Radford (2003) on semiotic 'objectification', as does Radford's (2008a) own paper in this issue. Roth and Thom (2008) add to this an explicit case of the learning experience as an integration of multi-modal sensual perceptions, particularly sight and manipulations (but also verbalisation). Additionally, Nemirovski and Ferrara (2008) illustrate the phenomena of 'juxtaposition of displacements/ cubist composition' and 'articulation of disjoint cases' in learners' articulations of imagination.

Thus, this Special Issue offers a rich and varied collection of theoretical and grounded illustrations from which to generalise, and the reader should conclude that, despite the sceptic's doubt, gestures are crucial to mathematics or at least to some aspects of mathematics education. Only perhaps in the limited variation in contexts of illustration might doubt still linger.

The contexts of mathematics education used to illustrate and embed the theoretical and conceptual discussions are varied, including graphing of advanced functions (Arzarello et al. 2008), graphs and motion sensors in high school (Radford 2008a), coordinate representations of space using lasers in a bilingual high school class (Nemirovski \& Ferrara 2008), teachers' discussion of fractions (Edwards 2008), visualisation of plane cross sections of the imaginary pyramid (Maschietto \& Bartolini Bussi 2008) and infant experience of three-dimensional solids (Roth \& Thom 2008). It is immediately apparent that most of these contexts are inherently spatial, as has been most of the previous study of gestures (e.g. Roth \& Bowen 2003, on graphs). Even in the case of fractions, the gestures mostly highlight the spatial embodied aspects of the fraction models being discussed (e.g. cutting of the imaginary fraction cake). One notices similarly in Radford's previous studies of gestures involving algebra and the generalisation of the variable conception, the gestural analysis focusses our attention on a spatial sequentiality embodied in a physical model (thus, the variable has been represented in what Nemirovski and Ferrara (2008) might call a kind of 'cubist' form in this model, see Radford 2003).

Might one conclude then that gesture is important when essential aspects of the mathematics are spatially organised? And is this principle more true of some mathematics than others or of some mathematical styles and some mathematicians than others? Or might we conclude that - if gestures are important to learning - then spatial organisations, representations and models are vital, at least in the early stages of learning new mathematics? Finally, might we also conclude that gestural modalities of communication are important not only in showing how mathematics can be learnt but also in understanding the critical mediating role of spatially organised models in this learning process?

\section{Understanding the meaning of gestures and models in learning activity}

In many cases where embodied learning processes are illustrated, a physical model of some kind is used as a pedagogical tool to mediate the learner's construction of mathematics: number lines, patterns of spatially arranged dots, double-number line or abacus models, etc. I have, with colleagues, illustrated the use of some of these in (1) analyses of studentworker communication in the workplace (Williams \& Wake 2007a, b) and (2) analyses of learning trajectories in teaching experiments akin to that of Maschietto and Bartolini Bussi (2008), such as in the teaching of negative integers and strategies for two-digit subtraction 
(Koukkoufis \& Williams 2006; Linchevski \& Williams 1999; Williams, Linchevski \& Kutscher 2008b). I will draw on two of these experiences now to add to the progress made in this volume and to draw attention to two important conceptions not much discussed in the six papers.

First, consider the case of the double abacus as a tool for representing positive and negative integers when teaching addition and subtraction (see Fig. 1). This apparatus is designed to allow multiple representations of integers but simultaneously to record the scores of two teams in various games, thus providing a model of a real-game situation and at the same time a model for the integers to be constructed. Essentially, the integer is represented by the subtraction, i.e. the signed difference, of the ordered pair of counting numbers of beads on the two columns - so Fig. 1 shows the pair $(8,10)$ and represents the integer -2 . Of course, the same integer value or score would be given by two piles of beads of height 7 and 9 , i.e. $(7,9)$. A mathematical analysis of the integers leads one to conclude that $(8,10)$ is just one instance of the equivalence class of similar ordered pairs $\{(x, y): x=y$ $-2 ; x$ and $y \geq 0\}$ that 'defines' the integer, -2 , and that allows the addition and subtraction schemes for whole numbers to be extended to the whole domain of integers (i.e. positive and negative numbers). Thus, one can take away -10 , say, from -2 by physically taking away ten from the 'negative column'-but this is only physically possible if the selected representation has at least ten of the minus beads on the negative column on the right-hand side of Fig. 1.

In our teaching experiment, the critical observation that constructs or objectifies an integer conception for the first time is, we argue, the observation that $(4,6),(8,10)$, etc. all have the same numerical 'value' in some sense that allows them to be ordered, for instance,

Fig. 1 The double abacus consists of two piles of beads or cubes, here representing -2 as $(8,10)$

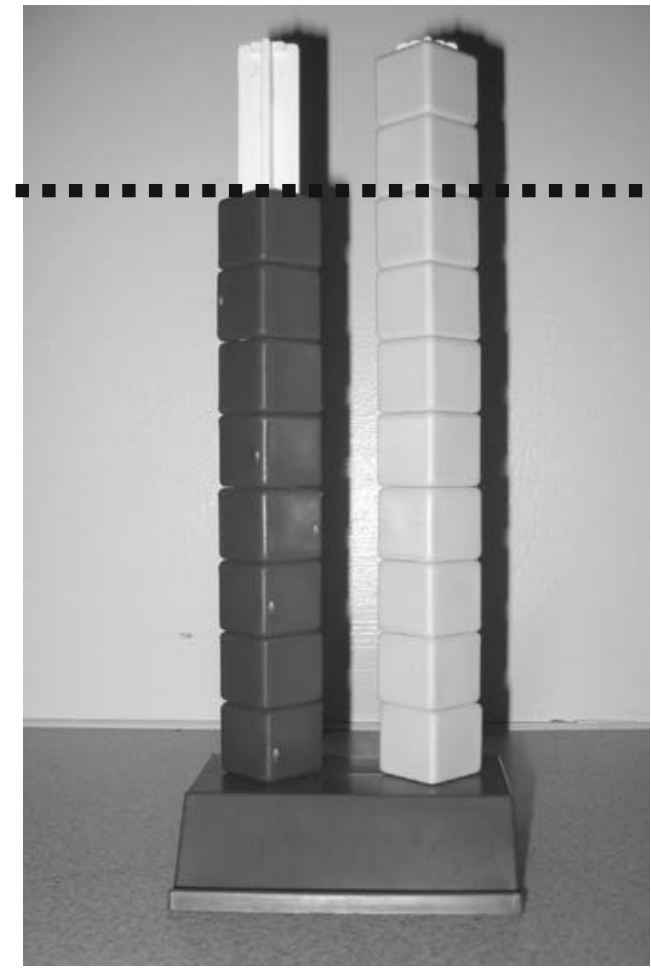


when the children say that $(4,6)$ and $(8,10)$ both represent the same 'score' for their opposing team of two points. This might be, and usually was, achieved by the children counting the 8 , counting the 10 and reasoning that the ten beads were 'points for them': the 8 being 'points for us', so 'they are two ahead of us' or the score is 'two to them'. In the context of this game, the meanings of $(8,10)$ and $(10,8)$ are clearly and intuitively different, even though the semiotic bundles of 'score', gesture, abacus, and the pairs of numbers are very close and potentially confused.

However, in our classroom research, a key 'objectification' arose when this reasoning was abbreviated by the action of attending to, or operating on, the gap (difference) between the two piles of beads - notably by gesturing with a cutting gesture at the horizontal plane at the top of the lower of the two piles of beads (see dotted line in Fig. 1). This cutting gesture is more or less isomorphic to the 'sawing' gesture of the children indicating a plane-pyramidal section in Maschietto and Bartolini Bussi (2008) - but in our case it arises as an inhibited action. That is, usually (not always) the action of separating the top few 'extra' beads to be counted would come before being signified by the gesture. It is also intuitive to signal the value of the score by the magnitude of the gap between the two piles, e.g. using the "C-shape" gesture isomorphic to that of Fig. 6a in Edwards (2008) and Fig. 7 of Arzarello et al. (2008). The two branches of the C-gesture simply indicate an interval or gap between the two points (or lines) indexed by thumb and forefinger.

The meaning of the gestures in different contexts is distinct because the contextual configuration of these situations is quite different (Halliday \& Hasan 1985). All one can say in general is that the cut or saw indicates (and is somewhat iconic of) a plane, and the Cgesture marks out a gap or interval that defines some segment of space or of a line. In much the same way, we can ask 'what is the meaning of a pointing finger?'-it is simply a gestural action intended to draw or focus attention on the object indicated. As such, it is an objectification, but the meaning of this is only visible in the field of, or the contextual configuration of, the communication (here, we apply the sociolinguistic term as if gesture was the same as language or text). Thus, not only is multimodality implicated, so is the whole context of activity: in our case, this included the notion of the 'team game scoring' that had been imported from children's prior experience of similar games, as well as the materials at hand, and the rules and language introduced by the 'teacher-researcher'.

The observation that the very same gesture has completely different meanings should be obvious. It is essentially no different from the observation that the meaning of language is generally found in context (shouting 'fire' means one thing in a crowded theatre and another in front of the firing squad). Therefore, my point is that if 'meaning' is the key unit of analysis, then not only is the analysis of the gesture inadequate but so is the 'semiotic bundle'. The analysis of meaning, according to Activity Theory, not only requires an understanding of the tool-mediated action itself (e.g. the utterance that contains the speech-gesture-inscription), it also requires an analysis at the level of the social activity, i.e. the collective, object-orientated activity: not just its mediating artefacts or tools, but also its 'object', rules, interactional norms and division of labour. Indeed, in many cases, a particular system of activity is not in itself sufficient to understand the context, and the boundary between at least two systems might need to be included in the analysis (Leont'ev 1978; see also Engeström \& Cole 1997; and in the mathematics context Williams \& Wake 2007a, Williams, Black, Hernandez-Martinez, Davis, Pampaka \& Wake 2008; Williams 2008).

Arzarello et al. (2008) begin to address this dimension when they describe the 'semiotic game': the teacher adopts the C-signs spontaneously produced by some of the students in his own mathematical utterances, thus scaffolding new mathematics and interweaving it with the students' own spontaneous gestures. In such an activity, the teacher-implicitly or 
explicitly-mediates for the students: it is not clear that all teachers see their role in teaching and learning activity in quite this way, and so another teacher might adopt an instructor role in which this teaching behaviour might not be seen as efficacious. These two distinct roles - the 'mediating teacher' and the 'transmissionist-instructor' - are in fact cultural-historical constructs (see Williams 2008). I speculate that the mediating teacher seeks to scaffold mathematics by working with the intuitive spontaneous gestures of the learner because they have identified themselves and their practice in some kind of Vygotskyan framework. In a similar vein, one can easily imagine that a 'mediating teacher', in the context of the integer games, might recognise the significance of the learner's C-sign and formalise the use of two types of C-signs for this purpose, with the thumb below the forefinger for positive amounts and its rotation with the thumb above the forefinger for negative amounts.

Consider another of our teaching experiments where young children are persuaded to play a kind of shopping game whereby they can purchase various desirable goods using play money, which consists of cards cut into 'tens' (strips marked out with ten 'ones') and 'ones' (which are just one tenth the length of the 'ten' strip). Typically, when called upon to pay an amount, say $\$ 28$, a child may have a number of tens but insufficient ones and may be stuck.

In one episode, a child in such a quandary pauses for a long while, then tentatively holds out three ten strips, and the other children in the group say something like "yes - and she will have to give you change back" and they then collectively set about working out this change (Williams et al. 2008).

Regarding the child's gesture-action of tentatively 'holding out the 30', we and the children present understood this in a certain way, as a hesitant offer of payment. We drew on our shared understanding of the culture of 'shopping' and the social rules involved: the purchaser is not obliged to pay the exact amount, but there is instead an obligation on the shopkeeper to provide some change. So why the hesitation? Perhaps, for one thing, this is all happening in school and it is not 'really' in a shop: the rules of schooling have been shaped by the special, very distinct cultural history of 'schooling', of course, and the series of social obligations implied by 'shopping' needs to be reconstructed therein. The context of this shopping simulation activity is thus at the border between 'schooling' and 'shopping': this affords the manipulation of the rules in apparently 'arbitrary' ways that would not be allowed in the real practice of shopping (particularly, one notes that in school the teacher gets to rewrite the rules) but encourages intuitive appeals, usually semihumorously, to knowledge and practices from the outside-school activity referenced.

This strategy of payment was designed by the researcher-instructors to provide an intuitive strategy on which a 'payback' method of subtraction would be built: thus, subtracting 28 from what the child had in the wallet - say 53 - when the 53 has insufficient 'ones' to pay the 8 is achieved by paying 30 from the 50 (leaving 20) and 'paying back' 2 to the three tens (final total in wallet: $20+3$ plus the 'paid back' 2 makes 25 ). We therefore saw this 'offering payment' gesture as a kind of germ of the later 'payback' subtraction strategy, similar to the way the cutting or sawing gesture objectifies the integer 'score' on the double abacus mentioned hitherto. In both cases, we believe the context of activity (shopping, scoring the game) was essential in making these objectifications meaningful.

I have mentioned two key 'moments' in two teaching experiments, when a gesture or an action provided the first objectification of a key idea or strategy that eventually may grow if carefully nurtured and tended, like a mathematical germ or seed, into new mathematics. It may be that Kenya (described by Roth \& Thom 2008) is similarly experiencing such a 'threshold' moment when she decides to place her cylinder with the other 'tubes' and thus 
names her experience of the shape. In fact, it may be that such moments are researchers' favourites for analysis - we cherry pick such moments of creativity because we believe they are critical 'threshold' moments in learning trajectories ${ }^{3}$.

\section{The threshold moment in learning}

Radford (2008a) points out that when the learner has become expert, his or her need for gestures etc. may fall away. Somehow, the moment of 'dawning of truth' or the flash of insight - what I will here call the 'threshold moment' when one somehow crosses the threshold and sees something important differently-is rarely mediated verbally. This seems to be as true of professional mathematicians as it is of the rest of us, as accounts from mathematicians and scientists in Koestler (1964) suggest. Thus, Poincare is said to have remarked about the discovery of the Fuchsian functions; their existence came upon him all of a sudden, at the very moment, he recalled, when he put his foot on the bus in Coutances, whereupon he knew instantly and with certainty that he would be able to produce the required proofs routinely later 'at his leisure' (Hadamard 1945).

But what is involved in this moment, when the learner crosses the threshold? One answer is the carrying across of (or interaction between) what is obvious in one domain into the new domain of interest, the essence of metaphor or modelling. These involve intuitions in the source domain revealing new insights in the target domain: many if not all of these are spatial and hence 'obvious' when 'seen' or 'grasped'. This embodied language is not accidental - the role of gesture and sight seems often to be the key to the suddenness of the 'flash'.

To the question, can all mathematical learning be like this, the answer for each instance of mathematics can only be empirical, and poses a further question, 'Can we design learning trajectories like this for all important mathematics'? In trying to make arbitrary strings of digits meaningful, the mnemonist may adopt strategies such as giving each digit a colour and then narrating a striking story in which the author takes a familiar and welltrodden walk while meeting objects of the required colour in some order: "I went out of my front door and tripped over a giant cucumber (green=3) walked past the post box $($ red=1) and luckily passed the neighbours black $(=4)$ cat $\ldots$ etc." Thus, an already familiar space and a striking narrative provide the narrator with an imagined path to travel through that models the digit string. The 'cubist' placing of facets of the concept in sequence here makes use of the narrative predisposition of our culture (Bruner 1996).

But mathematics does not normally have to be learnt mnemonically, as there are so many spatial and cultural precursors for building up mathematics with meaning — at least this is true of early mathematics (Lakoff \& Nunez 2000). One of the key purposes of instructional design should be to achieve this and so to help learners achieve meaningful mathematics in practice.

\footnotetext{
${ }^{3}$ An aside on the term 'threshold'. A small but growing literature on threshold concepts in Higher Education suggests that each academic discipline has certain characterising threshold concepts that serve to 'identify' those who see themselves as belonging to the relevant cognate academic community (Meyer \& Land 2006). These concepts are typically troublesome for learners and teachers but, once the light has dawned, provide a new view of the discipline-episteme as well as the self. Most of this work has been in subjects such as economics and also geology, and not much has been done in mathematics from this point of view, though it would seem that 'proof' and 'infinitesimal calculus' provide possible candidate concepts. However, the 'threshold' metaphor seems apt to the moments discussed here; other terms such as 'germ cell' and 'growth point' have too technical a meaning already defined to use or abuse them in this way.
} 


\section{Conclusion: the universality of embodiment in mathematics and modelling}

It might be trite to point out that all thinking is mediated by neural substrates and is therefore material and so embodied. But then experience of pure contemplation is itself sensuous: and, according to the Kama Sutra, solving mathematics problems should enhance the erotic experience by exciting one's lover's intellect: what could be more embodied than that? But the more interesting question would seem to be: is there a universal role for embodiment in the teaching and learning, and so pedagogy, of mathematics? What would be lost if classroom mathematical interaction was all verbally, or textually, mediated? Eros, viscera, emotion?

The summation of the arguments in the papers in this issue suggests that we would lose the spatial aspect of communicating with models, modelling, metaphor and analogy-whereby embodiment and gestures help learners engage with mathematics and so lose all the immediacy and meaningfulness of intuition that carries spatially between domains of knowledge and awareness. Kenya's 'feel' for the tube, Andrea's grasping of the decreasing magnitude of delta (as the thumb and forefinger of the C-sign close up) in the secant triangle, the student teacher JL's (Fig. 4 of Edwards 2008) cutting of the fraction pie to make equivalent fractions and so on, all engage the learner-subject's body in interaction with spatially organised models of mathematics (the cylinder, the graph of the function, the fraction pie). I have argued that to this we should add that these intuitions might carry meanings by virtue of the whole context or configuration of activity and not merely by virtue of its operational (and spatial) reference: I can only speculate (as I did with the 'mediating' teacher) in each of these situations how relevant that level of analysis might have been.

How universal is this and how universal could it be? I am reminded of the nursery class teacher who believed she was lucky to have a brand new school building to work in - but she arrived to find the nursery classroom had a computer and an electronic whiteboard but no sand pit. In a similar vein, recently a head teacher in a 'good' local primary school asked me (as the visiting maths expert) if I thought that manipulatives were out of date now that we have software for everything. In professional practice, then, it seems we may have our task cut out to preserve any embodied mathematics pedagogy, never mind promote its universality.

Nevertheless, if pressed I would suggest 'yes' to universality on theoretical grounds even though a diversity of empirical illustrations is no proof of universality. Theoretically, according to Peirce (1931-1958), induction and deduction do not offer us thresholds into essentially 'new' territory: that can only come through abduction or the carrying across of insights and intuitions from other domains (see Peirce 1931-1958, CP, vol. 2). Koestler (1964), too, argues that creativity involves simultaneous apprehension in the mind of two matrices of knowledge or experience, which is characteristic of metaphor and modelling. This 'carrying across' from domain to domain, rather than being only one way, usually resonates between the two (or more) domains. I suggest that this movement begs to be organised spatially because metaphor can always in principle be spatially, diagrammatically represented (if only minimally by using points for objects and lines for the 'carrying across' as if it were a function) and hence begs to be 'grasped', 'seen' and embodied (see Radford 2008b).

Yet the examples I have alluded to above may not be simply reduced to a diagrammatic representation of a mapping between two domains: the threshold moment when the intuition "here look I have this..." or "OK and she has to give you change" is brought to bear springs from the whole embodied social habitus invoked by shopping in the game not by a modelling diagram as such. I therefore ask for 'culture' to be viewed as part of the learners' embodied habitus'. 
The pedagogical agenda then is to find intuitive grounds in domains that can resonate with (new) mathematics and to provide spatial models for translating between domains that allow cultural resources to be translated or re-presented in the new mathematics. These models should be figurative and spatially organised and afford embodied communication, but the domain of experience offers meaning and intuition to mathematics also: what a wonderfully complicated world pedagogy inhabits.

\section{Afterword}

According to Mithen (1996), the cultural explosion of the human species occurred when art, religion, etc. emerged 'suddenly' 30-60,000 or so years ago, and this should be associated with the evolution of the modern human mind: this emerged from the invasion of different, hitherto separate, 'modules' that dealt with 'hand-tool' functioning, 'theory of mind', natural-history knowledge, etc. Thus, he claims, language drew on the memory systems that had evolved to store masses of information about nature, i.e. what is edible or poisonous, etc.: thus, the mind came to be capable of memorising a huge vocabulary quite suddenlyin evolutionary terms. Likewise, the capacity to manipulate the 'theory of mind' module emerges with group leadership: the leaders are not so much those that are fittest or strongest, but rather those that can manipulate other humans in the group for their own ends. Whilst not convinced of what Pinker (2002) calls the 'just so' stories of evolutionary psychologists, I find the notion that our tribal 'leaders' are not 'fit' but are rather 'smart' at manipulating others in their own interests empirically compelling (credit crunch billionaires and university managers spring to mind).

Although Mithen's original discipline was archaeology, his thesis draws on the literature from child development; this suggests the growth of the human mind involves processes of threading together of the functions of the body (our first manipulable 'tool' or instrument) with other modules, e.g. language and mathematics. (Note that this is not the same modular theory of perceptions that Arzarello et al. 2008 discuss). The Vygotskyan conception of the zone of proximal development suggests that culture is accessed by learners through social activity in which culture provides mediating tools and instruments for joint activity. The child's action of offering a payment is supported by others who say "that's right - and she must give you change"- - which is afforded by the 'shopping' cultural model or script.

The dog which takes his lead to his master's feet is interesting because his gesture thereby demonstrates his learning and cultural accomplishment, which presumably arose from their joint activity of 'walkies'. I have seen shepherds working with dogs in which a culturally-historically evolved and semiotically mediated joint activity of farming is practised (involving a quite sophisticated language of whistles etc.). It also seems arguable that the dog that needs the walk is expressing a subjective agency and is symbiotic with the human's in a joint activity, whose outcome is the satisfaction of their joint needs for recreation. This can be similarly seen when the recovering heart operation patient is given a pet to reduce stress (in preference to their spouse, say!).

The dog's 'voice' is clearly heard in our politics and society now-he is part of many families and makes demands on the budget in food and veterinary health care. A lot can be accomplished by non-verbal, embodied and gestural communication, then, though when we look for its meaning we always need to look to the larger social, activity system of culturally and socially mediated, and so at least partly human, joint activity. 


\section{References}

Arzarello, F., Paola, D., Robutti, O., \& Sabena, C. (2008). Gestures as semiotic resources in the mathematics classroom. Educational Studies in Mathematics, this issue doi:10.1007/s10649-008-9163-z.

Bruner, J. (1996). The culture of education. Cambridge: Harvard University Press.

Edwards, L. (2008). Gestures and conceptual integration in mathematical talk: Remembering and problem solving. Educational Studies in Mathematics, this issue doi:10.1007/s10649-008-9124-6.

Engeström, Y., \& Cole, M. (1997). Situated cognition in search for an agenda. In D. Kirshner \& J. A. Whitson (Eds.), Situated cognition: Social, semiotic, and psychological perspectives. Hillsdale: Lawrence Erlbaum.

Hadamard, J. (1945). The psychology of invention in the mathematical field. Princeton: University of Princeton Press.

Halliday, M. A. K., \& Hasan, R. (1985). Language, context and text: aspects of language in a social semiotic perspective. Oxford: Oxford University Press.

Koestler, A. (1964). The act of creation. London: Hutchinson.

Koukkoufis, A., \& Williams, J. (2006). Semiotic objectifications of the compensation strategy: En route to the reification of integers. Revista Latinoamericana de Investigación en Matemática Educativa (Numero Especial), 9, 157-175.

Lakoff, G., \& Nunez, R. (2000). Where mathematics comes from. NewYork: Basic Books.

Leont'ev, A. N. (1978). Activity, consciousness, and personality. Englewood Cliffs: Prentice Hall.

Linchevski, L., \& Williams, J. (1999). Using intuition from everyday life in 'filling' the gap in children's extension of their number concept to include the negative numbers. Educational Studies in Mathematics, 39(1-3), 131-147. doi:10.1023/A:1003726317920.

Maschietto, M., \& Bartolini Bussi, M. G. (2008). The potential of gestures as generalization devices: Visual pyramid in primary school. Educational Studies in Mathematics, this issue doi:10.1007/s10649-0089162-0.

Meyer, J. H. F., \& Land, R. (2006). Overcoming barriers to student learning: Threshold concepts and troublesome knowledge. London: Routledge.

Mithen, S. (1996). The prehistory of the mind: a search for the origins of art, religion and science. London: Thames and Hudson.

Nemirovsky, R., \& Ferrara, F. (2008). Mathematical imagination and embodied cognition. Educational Studies in Mathematics, this issue doi:10.1007/s10649-008-9150-4.

Peirce, C. S. (1931-1958). Collected papers (vol. 1-8). Cambridge: Harvard University Press.

Pinker, S. (2002). The blank slate. London: Penguin.

Radford, L. (2003). Gestures, speech, and the sprouting of signs. Mathematical Thinking and Learning, 5(1), 37-70. doi:10.1207/S15327833MTL0501 02.

Radford, L. (2008a). Why do gestures matter? Sensuous cognition and the palpability of mathematical meanings. Educational Studies in Mathematics, this issue doi:10.1007/s10649-008-9127-3.

Radford, L. (2008b). Diagrammatic thinking: Notes on Pierce's semiotics and epistemology. PNA, 3(1), 1-18.

Roth, W.-M., \& Bowen, G. M. (2003). When are graphs ten thousand words worth? An expert/expert study. Cognition and Instruction, 21, 429-473. doi:10.1207/s1532690xci2104_3.

Roth, W.-M., \& Thom, J. S. (2008). Bodily experience and mathematical conceptions: From classical views to a phenomenological reconceptualization. Educational Studies in Mathematics, this issue doi:10.1007/ s10649-008-9138-0.

Williams, J. S. (2008). The cultural historical mediation of a professional identity. Presented at ISCAR San Diego. http://www.lta.education.manchester.ac.uk/TLRP/iscar $\% 20$ the $\% 20$ cultural $\% 20$ historical $\% 20$ for mation $\% 20$ of $\% 20 \mathrm{a} \% 20$ professional $\% 20$ identity.pdf.

Williams, J. S., \& Wake, G. D. (2007a). Black boxes in workplace mathematics. Educational Studies in Mathematics, 64(3), 317-343. doi:10.1007/s10649-006-9039-z.

Williams, J. S., \& Wake, G. D. (2007b). Metaphors and models in translation between college and workplace mathematics. Educational Studies in Mathematics, 64(3), 345-371. doi:10.1007/s10649-006-9040-6.

Williams, J. S., Black, L., Hernandez-Martinez, P., Davis, P., Pampaka, M., \& Wake, G. D. (2008a). Repertoires of aspiration, narratives of identity, and cultural models of mathematics in practice. In M. César, \& K. Kumpulainen (Eds.), Social interactions in multicultural settings. Rotterdam: Sense Publishers.

Williams, J. S., Linchevski, L., \& Kutscher, B. (2008b). Situated intuition and activity theory fills the gap. In A. Watson, \& P. Winbourne (Eds.),New directions for situated cognition in mathematics education (pp. 153178). New York: Springer. 